\title{
Applying protective rectal tube in intestinal anastomosis
}

\author{
Abdolhassan Talaiezadeh, Mohammad-Hussein Sarmast Shoushtary, Amir-Ahmad Salmasi, \\ Maryam Avatefi Afkhami
}

Department of Surgery and Cancer Research Center, Ahvaz Jundishapur University of Medical Sciences, Ahvaz, Iran

Gastroenterology Rev 2018; 13 (2): 99-101

DOI: https://doi.org/10.5114/pg.2018.76006

Key words: ileostomy, rectal tube, rectal anastomosis.

Address for correspondence: Prof. Abdolhassan Talaiezadeh, Department of Surgery and Cancer Research Center, Ahvaz Jundishapur University of Medical Sciences, 6193673166 Ahvaz, Iran, phone: 989161184922 , e-mail: ahtalaiezadeh@yahoo.com

\begin{abstract}
Introduction: Colorectal resection is used for various diseases, including neoplasms (benign and malignant) and inflammatory bowel disorders. Leakage is one of the complications that increase mortality and morbidity in patients. The beneficial effects of a rectal tube in reducing anastomosis leak are controversial. Ileostomy as diverting stool may reduce risk of anastomosis leak.

Aim: To evaluate the results of rectal tube application to reduce leakage in low pelvic anastomosis.

Material and methods: In a prospective cohort study, 53 patients who were candidates for low anterior resection, extended low anterior resection, and ileal pouch-anal anastomosis, were enrolled in this study. After anastomosis, a large bore Foley was inserted transanally and put in the proximal of the anastomotic site, after fixation; we left it at least for 5 days then removed it if there was any sign of leakage.

Results: In this research, 53 patients were studied; 30 (56.6\%) were male and 23 (43\%) were female. Among 32 (60\%) patients who underwent total proctocolectomy and ileal pouch anal anastomosis, 24 (45\%) had ulcerative colitis and 8 (15\%) had familial adenomatous polyposis disorder. Among 21 (40\%) patients who had rectal cancer, 8 (15\%) cases had low anterior resection (LAR) and 13 (25\%) underwent extended LAR. Seventeen (32\%) patients used ileostomy and 36 (67.9\%) patients used a rectal tube. Anastomotic leak occurred in $6(11 \%)$ patients; all of them had ileostomy. No leakage or complication appeared in rectal tube cases. The difference was statistically significant $(p<0.001)$.

Conclusions: The results of our study suggest that using a rectal tube instead of protective ileostomy may reduce anastomosis leak in the lower pelvis by reducing intraluminal pressure to effect and lower tension at the anastomosis site in low pelvic intestinal anastomosis.
\end{abstract}

\section{Introduction}

Colorectal resection is used for various diseases, including neoplasms (benign and malignant), inflammatory bowel disease, and other benign conditions. Intestinal anastomotic leak is the most catastrophic complication in lower pelvis anastomosis like low and extended low anterior resection (LAR or extended LAR) in colorectal cancer or in lleoanal anastomosis in ileal pouch anal anastomosis (IPAA) in inflammatory bowel disease or familial adenomatous polyposis (FAP). Some papers have confirmed the beneficial effects of using rectal tubes [1, 2]. Transanal rectal tube placement for intraluminal decompression of the anastomosis site in LAR, extended LAR or IPAA is controversial among surgeons. Some surgeons believe that diverting stool through a protective ileostomy can help to reduce the risk of anastomosis leakage [3]; they use temporary ileostomy to support anastomosis that has a risk of leakage. Ileostomy complications include necrosis stoma, dehydration, and electrolyte imbalance. Parastoma-hernia and however ileostomy reduce the quality of life [4], so it is better to close it as soon as possible. However, the ileostomy closure has related side effects. We believe that putting a temporary transanal rectal tube in proximal of anastomotic site may cause sufficient intraluminal decompression to prevent or reduce the risk of anastomosis leak.

\section{Aim}

In this study we evaluate the results of applying a transanal rectal tube in lower chance of pelvic anastomosis leakage, instead of temporary ileostomy, in Ahvaz hospitals. 


\section{Material and methods}

In a prospective cohort study, 53 patients with lesions in the colon and rectum as rectal cancer and inflammatory bowel disease (IBD), who were admitted for LAR, extended LAR, or IPAA operations, were enrolled in the study. Patients in whom anal sphincters were involved or those who had incontinency preoperatively and so were not eligible for anastomosis were excluded from the study. Demographic information and any signs or symptoms of anastomosis leak and patients whom reoperated collected in Ahvaz hospital during 2011-2013.

Complications and anastomosis leak in patients who had protective ileostomy were compared with patients with a transanal rectal tube.

\section{Results}

In this research, 53 patients were studied; 30 (56.6\%) were male and 23 (43\%) were female. Among $32(60 \%)$ patients who underwent total proctocolectomy and IPAA 24 (45\%) had ulcerative colitis and 8 (15\%) had FAP. Among 21 (40\%) patients who had rectal cancer, 8 (15\%) cases recieved LAR and 13 (25\%) underwent extended LAR.

Seventeen (32\%) patients used ileostomy and 36 (67.9\%) patients used a rectal tube. Anastomotic leak occurred in 6 (11\%) patients; all of them had ileostomy. Leakage did not occur in any of the patients with rectal tubes. The difference was statistically significant $(p<0.001)$ (Table I).

Overall hospital admissions in all patients were 7 days; in patients with ileostomy and leakage were 21 days but in patients without leakage they were 6 days.
No leakage occurred in the rectal tube group. Hospital admissions in the rectal tube group were 6 days. No reoperation was done in our patients. Sixteen (28/3\%) of the 21 (40\%) patients with rectal cancer underwent neoadjuvant chemoradiotherapy, 14 (22/6\%) underwent extended LAR, and 2 (3/7\%) underwent LAR.

\section{Discussion}

Anastomosis leak as a major complication of colorectal surgery can increase morbidity, mortality, and chance of reoperation with prolonged hospitalisation [5]. The rate of anastomosis leak is reported as 2.9$12 \%$. This wide range of difference may be due to different definition of leak of anastomosis. The International Rectal Cancer Group offers a standard definition of leak anastomosis based on clinical symptoms: anastomosis leak is a lesion of the intestinal wall, leading to a loss of communication between the inside and outside of the lumen. Leak of anastomosis is divided into three categories according to severity: the first group that do not require any intervention, the second group that require medical intervention without the need of re-laparotomy, and the third group that require re-laparotomy [3]. Ileostomy is used as a technique that diverts the transit of stool before the anastomosis to reduce the leakage of anastomosis. There is controversy in using colostomy or ileostomy as diverting protective stoma.

Although diverting ileostomy is recommended, there is little data to recommend proximal colostomy as a diverting protective procedure [4]. Ileostomy closure can be done 8-12 weeks after LAR operation. This time is enough for healing of anastomosis and reduction of inflammation or adhesions of primary operation. Recent studies suggest ileostomy closure 2 weeks after primary

Table I. Results of insertion of transanal rectal tube and protective ileostomy in LAR, extended LAR, and IPAA operations

\begin{tabular}{|c|c|c|c|c|}
\hline \multirow[t]{2}{*}{ Parameter } & & \multicolumn{2}{|c|}{ Intervention } & \multirow[t]{2}{*}{ Patient $(n)$} \\
\hline & & Rectal tube & Protective ileostomy & \\
\hline \multicolumn{2}{|l|}{ Number of patients } & $36(67.9 \%)$ & $17(32 \%)$ & 53 \\
\hline \multirow[t]{3}{*}{ Type of surgery } & Extended low anterior resection (ELAR) & $13(25 \%)$ & 0 & 13 \\
\hline & Low anterior resection (LAR) & $8(15 \%)$ & 0 & 8 \\
\hline & Ileal pouch anal anastomosis (IPAA) & $15(28.3 \%)$ & $17(32 \%)$ & 32 \\
\hline \multirow[t]{5}{*}{ Underlying disease } & Ulcerative colitis & $9(16.9 \%)$ & $15(28.3 \%)$ & 24 \\
\hline & Rectal cancer & $21(40 \%)$ & 0 & 21 \\
\hline & Familial adenomatous polyposis (FAP) & $6(11.3 \%)$ & $2(3.7 \%)$ & 8 \\
\hline & Leakage & 0 & $6(11 \%)$ & 6 \\
\hline & Need for further surgery & 0 & 0 & 0 \\
\hline
\end{tabular}


operation, showing that there is a relationship between prolonged ileostomy and complications [5].

lleostomy is a simple procedure, but according to some papers its rate of complications is about $21-70 \%$. lleostomy should be closed as soon as possible due to reduced quality of life and complications [6].

In September 2008 Chude et al., in a study of 256 patients undergoing low anterior resection, performed ileostomy in 136 patients, but 120 patients were without ileostomy. They were monitored closely after surgery for anastomotic leak and all the problems and complications were recorded. This study concluded that the use of ileostomy in patients with rectal surgery is useful for reducing mortality and reducing the likelihood of anastomosis leak [7]. Okoshi et al. studied 25 patients with leakage from anastomosis after low anterior resection with double-stapler technique of the laparoscopic method. The leak was detected by radiological studies and digital exam; transrectal drainage was applied to large and unstable abscess. Nine of 25 patients required emergency surgery; 16 patients were treated conservatively, 12 patients were treated with transanal drainage, which was successful in 10 patients, and two patients required to ileostomy because of fistula formation with other organs. Average drain exposure time was 10-45 days. The study showed that transanal drainage is adequate for the treatment of lake anastomosis after LAR [1]. Xiao et al. in a study of 398 patients who underwent low anterior resection, divided them into two groups; one group with rectal tube placed in the rectum, and the other in which a rectal tube was not used. In patients with rectal tube and in patients without rectal tube, the leakage rate was $4 \%$ and $6.9 \%$, respectively. Overall, the study concluded that the use of the rectal tube is an effective method in reducing anastomotic leak [2].

The results of our study suggest the use of a rectal tube instead of protective ileostomy for reducing the leakage of lower pelvic anastomosis. According to our study, anastomotic leakage was not seen among procedures with a rectal tube. In laparoscopic surgery we can remove colon and insert a rectal tube for protection of anastomosis transannally [8]. Sixteen of 21 patients with rectal cancer underwent neoadjuvant chemoradiotherapy, and in all cases of rectal cancer rectal tubes were used to prevent leakage.

Limitation: The low sample size and single-centre nature of the study were the main limitations.

\section{Conclusions}

The results of our study suggest that use of a rectal tube instead of protective ileostomy may reduce the chance of anastomosis leak in the lower pelvis anastomosis by reducing the intraluminal pressure effect.

\section{Acknowledgments}

This paper is according to proposal CRC-9104 supported by the Cancer Research centre affiliated to Ahvaz Jundishapur University of Medical Sciences. The authors would like to thank the patients who assisted us in this research. This study was supported by the research fair of Ahvaz Jundishapur University of Medical Sciences.

\section{Conflict of interest}

The authors declare no conflict of interest.

\section{References}

1. Okoshi K, Masano Y, Hasegawa S, et al. Efficacy of transanal drainage for anastomotic leakage after laparoscopic low anterior resection of the rectum. Asian J Endosc Surg 2013; 6: 90-5.

2. Xiao L, Zhang WB, Jiang PC, et al. Can transanal tube placement after anterior resection for rectal carcinoma reduce anastomotic leakage rate? A single-institution prospective randomized study. World I Surg 2011; 35: 1367-77.

3. Essani R, Bergamaschi R. Anastomotic leak in colorectal surgery: a review. Gastroenterol Pol 2009; 16: 123-7.

4. El-Hussuna A, Lauritsen M, Bülow S. Relatively high incidence of complications after loop ileostomy reversal. Dan Med J 2012; 59: A4517.

5. Yeh CY, Changchien CR, Wang JY, et al. Pelvic drainage and other risk factors for leakage after elective anterior resection in rectal cancer patients. Ann Surg 2005; 241: 9-13.

6. Shabbir J, Britton DC. Stoma complications: a literature over view. Colorectal Dis 2010; 12: 958-64.

7. Chude GG, Rayate NV, Patris V, et al. Defunctioning loop ileostomy with low anterior resection for distal rectal cancer: should we make an ileostomy as a routine procedure? A prospective randomized study. Hepatogastroenterology 2008; 55 : 1562-7.

8. Kayaalp C, Yagci MA, Soyer V. Laparoscopic and natural orifice transluminal restorative proctocolectomy: no abdominal incision for specimen extraction or ileostomy. Videosurgery Miniinv 2016; 11: 115-20.

Received: 7.08.2016

Accepted: 20.06.2017 